\title{
O Sistemismo de Bunge como Instrumento Metodológico para Aprendizagem Ativa
}

\author{
Bunge's Systemic Approach as a Methodological Tool \\ for Active Learning
}

iD

Luan Moreira

${ }^{1}$ Instituto Federal de Educação, Ciência e Tecnologia de Mato Grosso do Sul (IFMS), Aquidauana, MS, Brasil. E-mail: luan.moreira@ifms.edu.br

Resumo: As pesquisas sobre Aprendizagem Ativa vêm compartilhando evidências de que, quando os estudantes são reunidos para resolução de problemas em um ambiente cooperativo com incentivos bem estruturados, o aprendizado é maximizado. No entanto, existe um grande desafio ao gestor da aprendizagem em relação à concepção e validação de modelos pedagógicos, pois há uma lacuna quanto à disponibilidade de uma metodologia para investigar a complexidade das relações sociais nos ambientes de aprendizagem. Neste trabalho propôs-se um instrumento metodológico para a Aprendizagem Ativa adaptandose o Sistemismo de Mario Bunge à prática pedagógica. Os resultados mostraram que, com a aplicação das regras metodológicas necessárias ao Modelo Composition, Environment, Structure and Mechanism (CESM), obteve-se maior objetividade na avaliação do modelo pedagógico idealizado pelo docente e possibilidade de aperfeiçoamento constante do mesmo, o que corroborou para a maximização da aprendizagem cognitiva dos estudantes envolvidos no estudo de caso.

Palavras-chave: Processo de ensino-aprendizagem; Modelo pedagógico; Ensino baseado em evidências; Sistemas complexos; Métodos de aprendizagem.

Abstract: Research on Active Learning has been sharing evidence that when students are brought together to solve problems in a cooperative environment with well-structured incentives, learning is maximized. However, there is great challenge for the manager of learning concerning the design and validation of pedagogical models because there is a gap regarding the availability of a methodology to investigate the complexity of social relationships in learning environments. In this paper, a methodological instrument for Active Learning has been proposed, by adapting Mario Bunge's Systemism to pedagogical practice. Results show that, with the application of the methodological rules required by the Composition, Environment, Structure and Mechanism (CESM) model, greater objectivity was obtained in the assessment of the pedagogical model idealized by the teacher as well as the possibility of constant improvement thereof, which contributed to the maximization of the cognitive learning by the students involved in the case study.

Keywords: Teaching-learning process; Pedagogical model; Evidence-based teaching; Complex systems; Learning methods. 


\section{Introdução}

A complexidade do mundo do trabalho das próximas décadas demanda que os cidadãos sejam protagonistas de sua própria aprendizagem, onde cada indivíduo não é mais um mero espectador passivo, mas sim, um agente ativo no processo de inovação e geração de valor.

Não é possível fornecer definições universalmente aceitas para todo o vocabulário de aprendizagem ativa, pois vários autores na área interpretaram alguns termos de formas diferentes. No entanto, é possível fornecer algumas definições geralmente aceitas. A aprendizagem ativa pode ser definida como um processo que exige que os alunos realizem atividades de aprendizagem significativas no ambiente de aprendizagem (sala de aula presencial e/ou digital) e pensem sobre o que estão fazendo (BONWELL; EISON, 1991; PRINCE, 2004).

Para verificar se a Aprendizagem Ativa maximiza o aprendizado dos estudantes, Freeman et al. (2014) realizaram uma meta-análise de 225 estudos que geraram dados sobre pontuações em exames e/ou taxas de reprovação, com o intuito de comparar o desempenho de alunos de cursos em Science, Technology, Engineering and Mathematics (STEM) sob aulas tradicionais versus aprendizado ativo. Os resultados apresentaram que a pontuação média nos exames melhorou em cerca de $6 \%$ nas sessões com aprendizagem ativa e os estudantes nas classes com aulas tradicionais obtiveram 1,5 vezes mais chances de falhar nos testes quando comparado com aqueles submetidos à aprendizagem ativa.

Deslauriers et al. (2019) também compararam a aprendizagem ativa e as aulas tradicionais. No entanto, o foco foi verificar a percepção auto relatada do aprendizado dos estudantes. Para tanto, sob condições controladas, separaram dois grandes grupos de estudantes em cursos introdutórios de física: (i) aprendizagem ativa (seguindo as melhores práticas para a unidade curricular) e (ii) aprendizagem passiva (palestras de instrutores experientes e altamente cotados). Ambos os grupos receberam o mesmo conteúdo, os alunos foram designados aleatoriamente e os instrutores não fizeram esforços para convencer os alunos sobre os supostos benefícios das estratégias. Os resultados mostraram que os estudantes obtiveram maior aprendizado nas salas de aula ativas, porém, quando analisada a auto percepção, os estudantes apresentaram inclinação positiva ao ambiente de aprendizado passivo.

Isso sugere que avaliar o aprendizado dos estudantes baseando-se apenas em suas respectivas percepções pode, inadvertidamente, conduzir à vieses cognitivos (levar os estudantes a desvios sistemáticos de lógica e a decisões irracionais).

Deslauriers et al. (2019) também enfatizaram a importância de preparar e treinar os alunos no início do semestre para a aprendizagem ativa e sugeriram que os docentes devem convencer os estudantes de que eles são beneficiados por essa abordagem. Sem essa iniciação, os alunos podem ser induzidos a um menor esforço cognitivo necessário para a aprendizagem ativa, que por sua vez, pode ter um impacto negativo em sua aprendizagem real. Esse fenômeno é especialmente importante para alunos que são iniciantes na aprendizagem ativa. 
Observa-se que a mera utilização de métodos e/ou estratégias de aprendizagem ativa não garante o aprendizado de todos os estudantes - que deveria ser o objetivo principal das instituições de ensino, conforme recomenda a Lei de Diretrizes e Bases (LDB), em seu inciso XIII, Art. $3^{\circ}$ e inciso III, Art. 13 (BRASIL, 1996) -, e que é necessária a realização de um processo de engajamento dos estudantes no início da jornada.

A desigualdade de aprendizagem entre grupos de uma mesma turma também é um aspecto relevante na gestão da aprendizagem. Lo e Hew (2017) sugeriram a elaboração do planejamento pedagógico focalizado nos alunos que demonstram menor desempenho. Assim, minimiza-se a diferença no aprendizado maximizando o desempenho dos estudantes mais fragilizados.

A Aprendizagem Ativa proporciona condições para o desenvolvimento de um ambiente colaborativo entre estudantes e professores. Além disso, a pluralidade de estratégias e métodos de ensino disponíveis possibilita que os gestores da aprendizagem dos estudantes personalizem os ambientes pedagógicos de acordo com os objetivos curriculares existentes. Para tanto, as instituições de ensino necessitam de professores com competências cognitivas e socioemocionais (UNESCO, 2017) relacionadas ao paradigma de revolução tecnológica atual, pois estes são os principais agentes facilitadores para a maximização do aprendizado dos estudantes (AKALIN; SUCUOGLU, 2015).

Nesse contexto inovador, e tendo em vista a importância do papel docente, a investigação científica das estratégias e métodos utilizados precisa se respaldar em um processo de conjecturas e refutações (POPPER, 2010). Em outras palavras, necessita-se de verificações empíricas da aprendizagem dos estudantes com o intuito de falsear, ou não, as hipóteses iniciais (estratégias e métodos adotados) sobre a condução do processo de aprendizagem.

No entanto, na literatura sobre Aprendizagem Ativa existe uma carência na disponibilidade de um arcabouço ontológico e metodológico que subsidie a modelagem (análise ex-ante) e a validação (análise ex-post) de estratégias e métodos de ensino e aprendizagem.

Para Bunge (2003) os problemas devem ser analisados de forma sistêmica, pois o tratamento dos elementos de forma isolada não é suficiente para problemas complexos. Outrossim, o sistemismo é uma proposta abrangente para resolução desses problemas.

O sistemismo tem dois componentes: ontológico e metodológico. O sistema ontológico afirma que o mundo é um sistema antes que uma coleção de indivíduos ou um bloco sólido. Seu parceiro metodológico é a visão de que os todos (wholes) são melhor entendidos por meio da análise (top-down) e indivíduos por meio da síntese (bottom-up). (BUNGE, 2006, p. 12-13, tradução e grifo nosso).

De acordo com Bunge (2000), o sistemismo fundamenta-se nos postulados a seguir.

I. Toda coisa, seja concreta ou abstrata, é um sistema ou um componente ou potencial componente de sistema.

II. Sistemas têm caraterísticas emergentes que seus componentes não possuem.

III. Todos os problemas deveriam ser abordados de forma sistêmica, em oposição às formas fragmentadas.

IV. Todas as ideias deveriam ser unidas em sistemas. 
V. O teste de qualquer coisa (como ideia, artefato, método) supõe a validade de outros itens que são tomados como pontos de referência provisoriamente.

Segundo Bunge (2003) todo sistema concreto pode ser descrito por meio de um modelo Composition, Environment, Structure and Mechanism (CESM):

- Composição (Composition): coleção de parte ou elementos componentes.

- Ambiente (Environment): coleção de itens que não fazem parte do sistema, mas atuam ou sofrem ação de algum componente.

- Estrutura (Structure): coleção de ligações entre componentes e entre esses e itens de ambiente.

- Mecanismo (Mechanism): coleção de processos que geram a novidade qualitativa. No Quadro 1 apresenta-se um exemplo aplicado de acordo com o modelo CESM.

Quadro 1 - Exemplo de aplicação do modelo CESM

\begin{tabular}{|c|c|c|c|c|}
\hline Sistema & Composição & Ambiente & \multicolumn{1}{c|}{ Estrutura } & \multicolumn{1}{c|}{ Mecanismo } \\
\hline Empresa & Funcionários e gerência & Mercado governo & $\begin{array}{l}\text { As relações de trabalho } \\
\text { entre membros da empresa } \\
\text { e entre esses e o ambiente } \\
\text { organizacional }\end{array}$ & $\begin{array}{l}\text { As atividades que resultam } \\
\text { em produtos e/ou serviços } \\
\text { da empresa }\end{array}$ \\
\hline
\end{tabular}

Fonte: adaptado de Bunge (2003).

Com exceção do universo, todos os sistemas possuem um ambiente e as ligações que são essenciais para sua respectiva auto-organização. Ademais, quanto maior o sistema, mais fracas serão as ligações.

A seguir são apresentadas as regras metodológicas que subsidiam a aplicação do CESM (BUNGE, 1997).

1. Dispor todo o fato social em seu contexto mais amplo.

2. Dividir cada sistema em sua composição, ambiente e estrutura.

3. Diferenciar os vários níveis de sistema e apontar suas relações.

4. Investigar os mecanismos que mantêm o sistema funcionando ou levam ao seu declínio.

5. Verificar se o mecanismo proposto tem coerência externa com o conhecimento produzido e, se necessário, verificar a hipótese experimentalmente.

6. Preferir hipóteses, teorias, explicações mecanísmicas às fenomenológicas e, por sua vez, preferir estas aos modelos de equilíbrio e às descrições de dados.

7. Caso haja um mau funcionamento do sistema, examinar todas as fontes do sistema e realizar a reparação alterando uma ou mais fontes.

\section{Objetivos}

Tendo em vista a lacuna, ontológica e metodológica, existente no processo de concepção, aplicação e verificação das estratégias e métodos de Aprendizagem Ativa, o objetivo deste trabalho foi desenvolver uma metodologia para o design de modelos pedagógicos baseados no Sistemismo de Mário Bunge, com intuito de contribuir para o processo de ensino e aprendizagem baseado em evidências. 


\section{Desenvolvimento}

\section{Modelo CESM aplicado à aprendizagem ativa}

De acordo com as regras metodológicas descritas anteriormente, elaborou-se uma versão adaptada à Aprendizagem Ativa, conforme apresenta-se no Quadro 2.

Quadro 2 - Regras metodológicas para elaboração de Modelo Pedagógico baseado no CESM

\begin{tabular}{|c|c|}
\hline Modelo CESM & Modelo CESM aplicado à aprendizagem ativa \\
\hline $\begin{array}{l}\text { 1. Dispor todo o fato social em seu } \\
\text { contexto mais amplo. }\end{array}$ & $\begin{array}{l}\text { 1. Dispor o processo pedagógico como unidade curricular, módulo, } \\
\text { projeto ou outro contexto, de acordo com o Projeto Pedagógico } \\
\text { do Curso. }\end{array}$ \\
\hline $\begin{array}{l}\text { 2. Dividir cada sistema em sua } \\
\text { composição, ambiente e estrutura. }\end{array}$ & $\begin{array}{l}\text { 2. Definir os elementos que compõem o sistema (alunos, professores, } \\
\text { tutores, técnicos etc.), o ambiente (sala de aula, Ambiente Virtual } \\
\text { de Aprendizagem, quadra poliesportiva etc.), e a estrutura (isto } \\
\text { é, as relações intersubjetivas e interobjetivas que ocorrem entre } \\
\text { os componentes do ambiente). }\end{array}$ \\
\hline $\begin{array}{l}\text { 3. Diferenciar os vários níveis de sistema } \\
\text { e apontar suas relações. }\end{array}$ & $\begin{array}{l}\text { 3. Conjecturar relações entre variáveis micropedagógicas } \\
\text { (aprendizagem cognitiva, socioemocional e comportamental) } \\
\text { e macropedagógicas (relações aluno-aluno e aluno-professor, } \\
\text { ambientes de aprendizagem, competências pedagógicas dos } \\
\text { docentes etc.). }\end{array}$ \\
\hline $\begin{array}{l}\text { 4. Investigar os mecanismos que mantêm } \\
\text { o sistema funcionando ou levam ao } \\
\text { seu declínio ou crescimento.. }\end{array}$ & $\begin{array}{l}\text { 4. Estabelecer a coleção de processos do mecanismo (ou seja, } \\
\text { estratégias e métodos de ensino, aprendizagem e avaliação). A } \\
\text { escolha das estratégias e métodos deve ser em consonância com } \\
\text { os objetivos de aprendizagem definidos no Projeto Pedagógico } \\
\text { de Curso (quando não disponíveis, deve-se defini-los por meio } \\
\text { de uma taxonomia adequada). } \\
\text { Estabelecer benchmark para avaliação do funcionamento do } \\
\text { sistema (desempenho em avaliações diagnósticas formativas e } \\
\text { sistemáticas, desigualdades de aprendizagem entre grupos etc.). }\end{array}$ \\
\hline $\begin{array}{l}\text { 5. Verificar se o mecanismo proposto tem } \\
\text { coerência externa com o conhecimento } \\
\text { produzido e, se necessário, verificar a } \\
\text { hipótese experimentalmente. }\end{array}$ & $\begin{array}{l}\text { 5. Verificar se as estratégias e métodos de ensino, aprendizagem e } \\
\text { avaliação são compatíveis com a produção acadêmica e, testar } \\
\text { empiricamente as hipóteses idealizadas em III e IV. }\end{array}$ \\
\hline $\begin{array}{l}\text { 6. Preferir hipóteses, teorias, explicações } \\
\text { mecanísmicas às fenomenológicas e, } \\
\text { por sua vez, preferir estas aos modelos } \\
\text { de equilíbrio e às descrições de dados. }\end{array}$ & $\begin{array}{l}\text { 6. Utilizar os instrumentos de avaliação em caráter processual } \\
\text { e cumulativo para a investigação da qualidade do modelo } \\
\text { pedagógico, tendo em vista o aprendizado individual de cada } \\
\text { estudante. É primordial estabelecer um espaçamento temporal } \\
\text { entre as avaliações da aprendizagem, pois o processo de } \\
\text { aprendizagem requer a formação de memórias de longo prazo } \\
\text { (BADDELEY; EYSENCK; ANDERSON, 2014; CARPENTER et al., 2012; } \\
\text { ROHRER; PASHLER, 2007). }\end{array}$ \\
\hline $\begin{array}{l}\text { 7. Caso haja um mau funcionamento } \\
\text { do sistema, examinar todas as fontes } \\
\text { do sistema e realizar a reparação } \\
\text { alterando uma ou mais fontes. }\end{array}$ & $\begin{array}{l}\text { 7. Se um ou mais resultados apresentados no benchmark são } \\
\text { insatisfatórios, deve-se realizar a correção do sistema antes de } \\
\text { um novo ciclo pedagógico, tendo em vista a melhoria contínua } \\
\text { do sistema e a busca pelo aprendizado de todos os estudantes. }\end{array}$ \\
\hline
\end{tabular}

Fonte: elaborado pelo autor.

\section{Estudo de caso de modelo pedagógico baseado no CESM}

No curso de Bacharelado em Engenharia Civil do Instituto Federal de Educação, Ciência e Tecnologia de Mato Grosso do Sul (2017) - IFMS -, câmpus Aquidauana, a matriz curricular apresentada no respectivo Projeto Pedagógico, está organizada em unidades curriculares, permitindo-sea análise sistêmica para cada unidade curricular. Em outras palavras, considerouse a Unidade Curricular como um sistema, os Estudantes e Docente Responsável como componentes, a Sala de Aula e o AmbienteVirtual de Ensino e Aprendizagem (AVEA) como itens 
do ambiente, e as Estratégias e Métodos de Ensino, Aprendizagem e Avaliação (Aprendizagem Baseada em Problemas, ABP, Sala de Aula Invertida, SAI, Avaliações Diagnósticas Formativas, ADF e Avaliações Diagnósticas Sistêmicas, ADS), como a coleção de processos do mecanismo. No Quadro 3 apresenta-se o modelo CESM aplicado estudo de caso deste trabalho.

Quadro 3 - Modelo CESM aplicado ao estudo de caso

\begin{tabular}{|c|l|l|l|c|}
\hline \multicolumn{1}{|c|}{ Sistema } & \multicolumn{1}{|c|}{ Composição } & \multicolumn{1}{|c|}{ Ambiente } & Estrutura & Mecanismo \\
\hline Unidade Curricular & Estudantes e Docente & Sala de Aula e AVEA & As relações intersubjetivas e & ABP \\
& Responsável & & interobjetivas entre estudantes & SAI \\
& & & e docente responsável, e entre & ADF \\
& & & esses e os itens do ambiente & ADS \\
\hline
\end{tabular}

Fonte: elaborado pelo autor.

A aprendizagem Baseada em Problemas ( $A B P)$, ou Problem-Based Learning ( $P B L)$, é uma estratégia de aprendizagem ativa que visa capacitar os estudantes a conduzir pesquisas, integrar teoria e prática, e aplicar conhecimentos e habilidades no projeto de soluções viáveis para problemas propostos (SAVERY, 2006). Também contribui para o desenvolvimento de pensamento crítico (ASYARI et al, 2016), atitudes positivas em relação aos cursos (DEMIREL; DAGYAR, 2016), motivação e capacidade de resolver problemas (CHIANG; LEE, 2016).

De acordo com EDUCAUSE (2012) a Sala de Aula Invertida (SAI), ou Flipped Classroom, é um método pedagógico no qual as práticas típicas propostas nos encontros presenciais são realizadas fora dos encontros presenciais. Em um ambiente de ensino e aprendizagem tradicional, o docente apresenta os conteúdos de forma expositiva e os alunos recebem passivamente exemplos de resolução de exercícios, seguidos por lições de casa. Em uma SAl, os alunos estudam o conteúdo da unidade curricular antes do encontro presencial e, em seguida, realizam atividades de aprendizagem individualmente ou em grupo durante o encontro presencial.

Para Moretto (2011) na Avaliação Diagnóstica Formativa (ADF) "[...] não é possível separar o avaliar do ensinar: avalia-se ensinando, ensina-se avaliando". O processo de avaliação se faz de forma contínua ao longo do período de ensino, com o intuito de se verificar a aprendizagem e, a partir da qualidade revelada, fazer o feedback imediato ao estudante de modo que este se torne consciente do seu progresso e assuma a responsabilidade pelo próprio aprendizado.

Na Avaliação Diagnóstica Sistemática (ADS) busca-se uma síntese sobre a qualidade da aprendizagem em determinados períodos, de acordo com o planejamento realizado (MORETTO, 2011). Essa avaliação tem por característica principal verificar se as estratégias e métodos de ensino e aprendizagem estão funcionando de acordo com o que foi planejado. Assim, de acordo com o diagnóstico realizado, pode-se corrigir eventuais não-conformidades e continuar com o processo de ensino e aprendizagem.

No modelo pedagógico idealizado, combinou-se a ABP e a SAI. Com a ABP esperavase que as interações sociais criassem as condições para o desenvolvimento em todos os domínios cognitivos (ver Quadro 5) devido às características emergentes que surgem de forma espontânea pelas interações entre os componentes (estudantes e docente). A SAI tem um caráter de dinamização da ABP, pois antecipa-se os objetos de conhecimento a serem trabalhados nos encontros presenciais, incentivando-se maior autonomia e responsabilidade dos estudantes pelo próprio aprendizado. No Quadro 4 e na Figura 1 são apresentadas as características do modelo pedagógico e seu escopo de execução, respectivamente. 
Quadro 4 - Estratégia de Ensino, Aprendizagem e Avaliação

\begin{tabular}{|c|l|l|}
\hline Etapa & \multicolumn{1}{|c|}{ Descrição } & \multicolumn{1}{c|}{ Características } \\
\hline 0 & Estudo Dirigido & $\begin{array}{l}\text { Etapa a distância dedicada ao compartilhamento, pelo AVEA, dos objetos de } \\
\text { conhecimento (em formato de e-book) que são trabalhados na etapa seguinte. O } \\
\text { envio ao AVEA é realizado com uma semana de antecedência à próxima etapa, com } \\
\text { o intuito de estimular o estudo antecipado por parte dos estudantes. }\end{array}$ \\
\hline 1 & $\begin{array}{l}\text { Avaliação da } \\
\text { Aprendizagem Coletiva } \\
\text { (AAC) }\end{array}$ & $\begin{array}{l}\text { Etapa presencial dedicada a diagnosticar o nível de aprendizagem potencial dos } \\
\text { estudantes. Trata-se da proposição de problemas (com complexidade ascendente) aos } \\
\text { estudantes de forma individual, mas com possiblidade de resolução em grupo. Esta } \\
\text { etapa é realizada no laboratório de informática, pois os problemas são submetidos } \\
\text { pelo AVEA. O docente atua como agente mediador da aprendizagem nessa etapa. }\end{array}$ \\
\hline 2 & $\begin{array}{l}\text { Feedback da AAC } \\
\text { (Avaliação Diagnóstica } \\
\text { Formativa) }\end{array}$ & $\begin{array}{l}\text { Etapa presencial dedicada a uma análise conjunta entre docente e discentes, } \\
\text { sobre a resolução da avaliação da etapa anterior. Trata-se de um momento para o } \\
\text { autodiagnostico do aprendizado de cada estudante, submetendo-os a um processo } \\
\text { crítico-reflexivo de seu desenvolvimento cognitivo. }\end{array}$ \\
\hline 3 & $\begin{array}{l}\text { Avaliação da } \\
\text { Aprendizagem } \\
\text { Individual (AAI) }\end{array}$ & $\begin{array}{l}\text { Etapa presencial dedicada a diagnosticar o nível de aprendizagem real dos estudantes. } \\
\text { Trata-se da aplicação de uma avaliação para resolução individual. }\end{array}$ \\
\hline 4 & $\begin{array}{l}\text { Feedback da AAI } \\
\text { (Avaliação Diagnóstica } \\
\text { Sistemática) }\end{array}$ & $\begin{array}{l}\text { Etapa presencial dedicada a uma análise conjunta entre docente e discentes, } \\
\text { sobre a resolução da avaliação da etapa anterior. Trata-se de um momento para } \\
\text { o autodiagnostico do aprendizado de cada estudante, focando-se nos domínios } \\
\text { cognitivos que apresentaram desempenho insatisfatório. }\end{array}$ \\
\hline 5 & $\begin{array}{l}\text { Avaliação de } \\
\text { Recuperação da } \\
\text { Aprendizagem (ARA) }\end{array}$ & $\begin{array}{l}\text { Etapa presencial dedicada a diagnosticar o nível de aprendizagem real dos estudantes } \\
\text { (com desempenho na etapa 3 inferior ao mínimo satisfatório) após o processo de } \\
\text { recuperação da aprendizagem realizado na etapa 4. }\end{array}$ \\
\hline
\end{tabular}

Fonte: elaborado pelo autor.

Figura 1 - Escopo de Execução do Modelo Pedagógico no Período 1
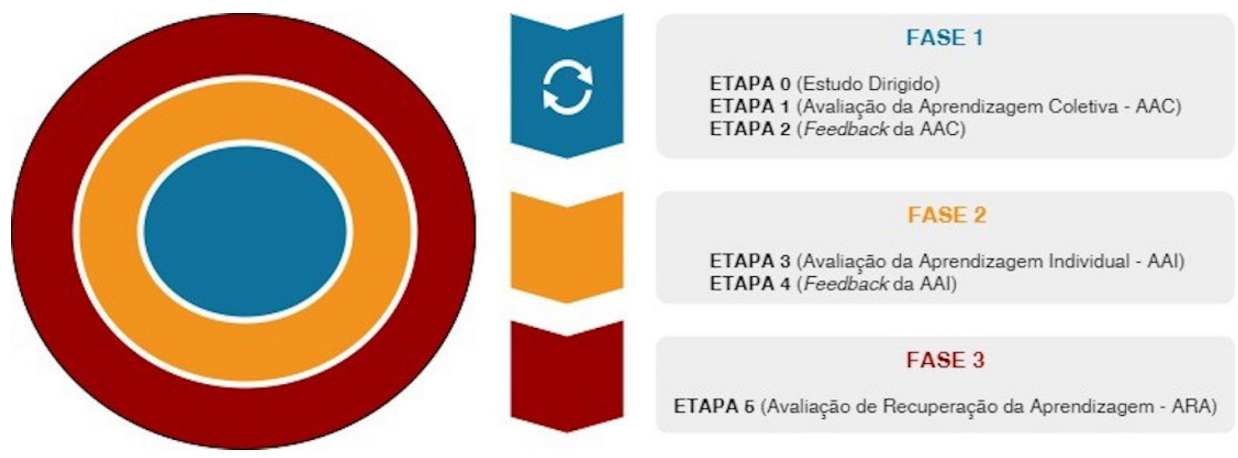

Fonte: elaborado pelo autor.

Observa-se na Figura 1 que a Fase 1 tem um caráter cíclico, ou seja, pode ser repetida conforme a necessidade do sistema. Também se enfatiza que a disposição das fases em círculos concêntricos denota um caráter evolucionista na aplicação de cada fase. Em outras palavras, a Fase 2 só se inicia após a conclusão da Fase 1 (transcendendo e incluindo as experiências obtidas na Fase 1) e a Fase 3 apenas ocorre após o término da Fase 2 (transcendendo e incluindo as experiências obtidas na Fase 2).

As avaliações diagnósticas das etapas 3 e 5 (Quadro 4) foram construídas a partir da Taxonomia Revisada de Bloom. Nesta são combinados o domínio cognitivo a ser desenvolvido e o processo cognitivo utilizado para a aquisição desse domínio (ANDERSON; KRATHWOHL, 2001). O domínio cognitivo é designado por substantivos e os processos cognitivos para atingi-los são descritos por verbos de ação, conforme apresenta-se no Quadro 5. Assim, pode-se verificar, empiricamente, o desenvolvimento de cada estudante em relação às atividades cognitivas da mais baixa ordem (conhecimento, entendimento, aplicação) e da mais alta ordem (análise, avaliação e criação). 
Quadro 5 - Taxonomia revisada de Bloom

\begin{tabular}{|c|c|c|c|c|}
\hline \multicolumn{2}{|l|}{ Hierarquia } & Domínio Cognitivo & Descrição & Verbos de Ação \\
\hline \multirow{3}{*}{$\begin{array}{l}\text { Atividades Cognitivas } \\
\text { da Mais Baixa Ordem }\end{array}$} & 1 & Conhecimento & $\begin{array}{l}\text { Recuperar conhecimentos } \\
\text { relevantes na memória de longo } \\
\text { prazo. }\end{array}$ & $\begin{array}{l}\text { Conhecer, Lembrar, Reconhecer, } \\
\text { Recordar. }\end{array}$ \\
\hline & 2 & Entendimento & $\begin{array}{l}\text { Construir significado a partir } \\
\text { de mensagens instrucionais, } \\
\text { incluindo comunicações oral, } \\
\text { escrita e gráfica. }\end{array}$ & $\begin{array}{l}\text { Entender, Interpretar, Exemplificar, } \\
\text { Classificar, Sumarizar, Inferir, } \\
\text { Comparar. }\end{array}$ \\
\hline & 3 & Aplicação & $\begin{array}{l}\text { Executar ou utilizar um } \\
\text { procedimento numa situação } \\
\text { dada, familiar ou não. }\end{array}$ & Aplicar, Executar, Implementar. \\
\hline \multirow[t]{3}{*}{$\begin{array}{l}\text { Atividades Cognitivas } \\
\text { da Mais Alta Ordem }\end{array}$} & 4 & Análise & $\begin{array}{l}\text { Separar a informação em suas } \\
\text { partes constituintes e detectar } \\
\text { como elas se relacionam umas } \\
\text { com as outras para formar a } \\
\text { estrutura e/ou propósito. }\end{array}$ & $\begin{array}{l}\text { Analisar, Diferenciar, Organizar, } \\
\text { Atribuir. }\end{array}$ \\
\hline & 5 & Avaliação & $\begin{array}{l}\text { Fazer julgamentos baseado em } \\
\text { critérios e padrões. }\end{array}$ & Avaliar, Checar, Criticar. \\
\hline & 6 & Criação & $\begin{array}{l}\text { Colocar os elementos em } \\
\text { conjunto para formar uma nova } \\
\text { estrutura com um todo coerente. }\end{array}$ & Criar, Gerar, Planejar, Produzir. \\
\hline
\end{tabular}

Fonte: Aranha, Santos e Garcia (2017).

O benchmark para verificar o funcionamento do sistema foi o desempenho médio ponderado de cada estudante $\geq 7,0$ (cada domínio cognitivo foi avaliado através de uma ou mais questões, quantificou-se o desempenho de 0 a 10, atribuiu-se um peso duas vezes maior para os domínios da mais alta ordem em relação aos da mais baixa ordem, e calculouse a média ponderada). Em caso de mau funcionamento do sistema (ou seja, 1 ou mais alunos não obtiveram desempenho satisfatório), utilizou-se a etapa 4 para recuperação do aprendizado e a etapa 5 para uma nova avaliação diagnóstica.

Ao final do processo, buscou-se aprimorar o sistema (modelo pedagógico) a partir de novas hipóteses que emergiram da investigação.

Na Figura 2 apresenta-se um resumo do processo de avaliação do modelo pedagógico.

Figura 2 - Escopo de Avaliação do Modelo Pedagógico

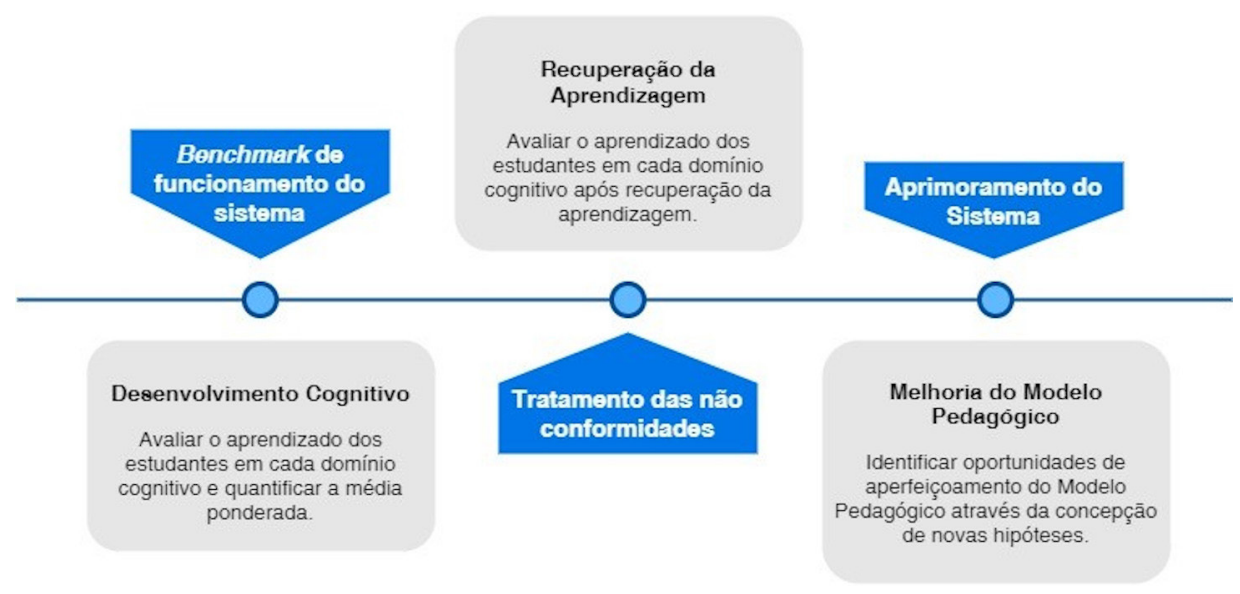

Fonte: elaborado pelo autor. 


\section{Análise dos Resultados}

O grupo analisado foi composto por 10 estudantes - total de alunos matriculados - da Unidade Curricular denominada Mecânica Geral oriunda do Curso Superior em Engenharia Civil, do IFMS, câmpus de Aquidauana.

Foram investigados dois períodos no semestre: Período 1 (02/09/2019 a 18/10/2019) e Período 2 (21/10/2019 a 06/12/2019).

\section{Período 1}

No Gráfico 1 apresenta-se o desempenho dos estudantes em cada domínio cognitivo de acordo com a Taxonomia de Bloom (Quadro 5). No Gráfico 2 mostra-se o desempenho médio ponderado, conforme explicado anteriormente.

Gráfico 1 - Desempenho nos domínios cognitivos no Período 1

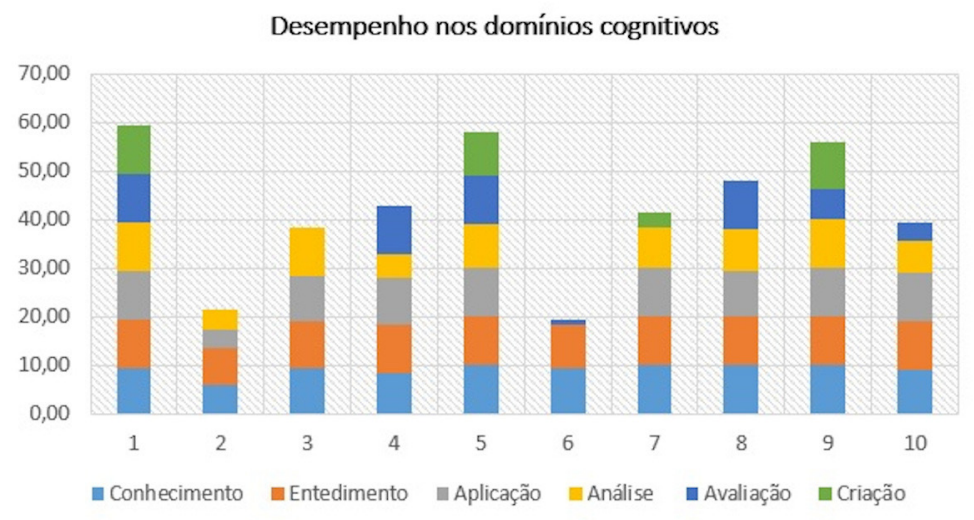

Fonte: elaborado pelo autor.

Gráfico 2 - Desempenho médio ponderado na Etapa 3/Quadro 4 do Período 1

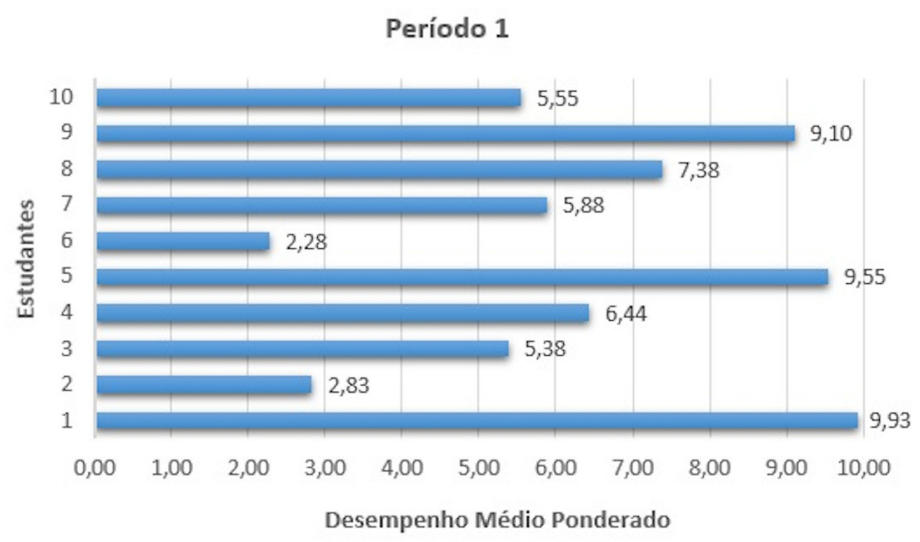

Fonte: elaborado pelo autor.

Observou-se que 6 estudantes não obtiveram o desempenho mínimo satisfatório de 7,0 (benchmark do sistema) e, entre estes, identificou-se baixos níveis de desenvolvimento nos domínios cognitivos da mais alta ordem, com especial atenção aos estudantes 2 e 6 que também apresentaram dificuldades no domínio cognitivo denominado aplicação. 
Também analisou-sea desigualdade deaprendizagem entreos grupos que representam a média dos $20 \%$ menores rendimentos e a média dos $20 \%$ maiores rendimentos. Constatouse uma desigualdade de $281,52 \%$, onde a mediana do desempenho médio ponderado foi de 6,16 .

Após a etapa 4 do Quadro 4 - que consistiu no processo de recuperação da aprendizagem - os estudantes com desempenho insatisfatório realizaram uma nova avaliação diagnóstica e os respectivos resultados estão expostos no Gráfico 3.

Gráfico 3 - Desempenho médio ponderado na Etapa 5/Quadro 4 do Período 1

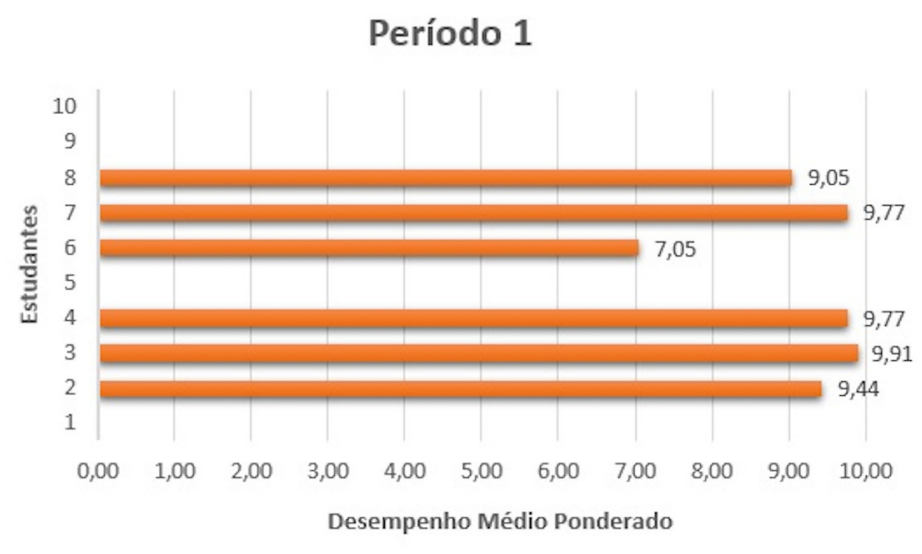

Fonte: elaborado pelo autor.

Observou-se que todos os estudantes que realizaram a Avaliação de Recuperação da Aprendizagem (Quadro 4) obtiveram melhoria no desempenho e, também, acima do mínimo satisfatório. A exceção foi o estudante 10 que optou por não fazê-la.

Também constatou-se que a desigualdade de aprendizagem entre os grupos foi de 57,5\% (redução de 79,58\%), onde a mediana do desempenho médio ponderado foi de 9,49 (aumento de 54,06\%).

Essas informações evidenciam que a estratégia de recuperação da aprendizagem foi eficiente para a correção do sistema, pois maximizou o desempenho cognitivo dos estudantes e também impactou positivamente na queda da desigualdade na aprendizagem entre grupos.

\section{Período 2}

A partir das não conformidades identificadas no sistema, as seguintes transformações foram realizadas para o Período 2:

- Criação da etapa Introdução aos Objetos de Conhecimento; e,

- Modificações na etapa 1 Avaliação da Aprendizagem Coletiva.

A adoção da etapa Introdução aos Objetos de Conhecimento foi realizada a partir de diálogos com os estudantes em relação à etapa de Estudo Dirigido, onde $90 \%$ dos estudantes relataram que não estavam estudando antecipadamente o material compartilhado. Com isso, ponderou-se que uma breve exposição dialogada poderia contribuir para uma aprendizagem mais significativa (Quadro 6, Etapa 1). 
Também se adicionou um segundo momento de avaliações diagnósticas coletivas (Quadro 6, Etapa 2), porém, com realização nos finais de semana. A motivação para isso foi a partir de relatos, da maioria dos estudantes, sobre a ausência de resolução de problemas complexos fora dos encontros presenciais.

No Quadro 6 apresentam-se as modificações em sublinhado e, na Figura 3, o novo escopo de execução do modelo pedagógico.

Quadro 6 - Estratégia de Ensino, Aprendizagem e Avaliação

\begin{tabular}{|c|c|c|}
\hline Etapa & Descrição & Características \\
\hline 0 & Estudo dirigido & $\begin{array}{l}\text { Etapa a distância dedicada ao compartilhamento, pelo AVEA, dos objetos de } \\
\text { conhecimento (em formato de e-book) que são trabalhados etapa seguinte. O envio } \\
\text { ao AVEA foi realizado com uma semana de antecedência à próxima etapa, com o intuito } \\
\text { de estimular o estudo antecipado por parte dos estudantes. }\end{array}$ \\
\hline 1 & $\begin{array}{l}\text { Iniciação aos Objetos de } \\
\underline{\text { Conhecimento }}\end{array}$ & $\begin{array}{l}\text { Etapa presencial dedicada a introduzir os estudantes aos objetos de conhecimento } \\
\text { da etapa anterior, por meio de uma breve exposição dialogada. O docente atua como } \\
\text { agente facilitador da aprendizagem nessa etapa. }\end{array}$ \\
\hline 2 & $\begin{array}{l}\text { Avaliação da Aprendizagem Coletiva } \\
\text { (AAC) }\end{array}$ & $\begin{array}{l}\text { Etapa presencial dedicada a diagnosticar o nível de aprendizagem potencial dos } \\
\text { estudantes. Trata-se da proposição de problemas (com complexidade ascendente) } \\
\text { aos estudantes de forma individual, mas com possiblidade de resolução em grupo. } \\
\text { Os problemas são submetidos pelo AVEA em dois momentos: presencial (laboratório } \\
\text { de informática) e a distância (sexta-feira com prazo para envio até segunda-feira). O } \\
\text { docente atua como agente mediador da aprendizagem nessa etapa, pois pode dialogar } \\
\underline{\text { com os estudantes, de forma individual, no momento presencial e pelo ambiente de }} \\
\text { comunicação assíncrona (grupo no whatsapp) no momento a distância. }\end{array}$ \\
\hline 3 & $\begin{array}{l}\text { Feedback da AAC (Avaliação } \\
\text { Diagnóstica Formativa) }\end{array}$ & $\begin{array}{l}\text { Etapa dedicada a uma análise conjunta entre docente e discentes, sobre a resolução } \\
\text { da avaliação da etapa anterior. Trata-se de um momento para o autodiagnostico do } \\
\text { aprendizado de cada estudante, submetendo-os a uma análise autocrítica e reflexiva. }\end{array}$ \\
\hline 4 & $\begin{array}{l}\text { Avaliação da Aprendizagem } \\
\text { Individual (AAI) }\end{array}$ & $\begin{array}{l}\text { Etapa dedicada a diagnosticar o nível de aprendizagem real dos estudantes. Trata-se } \\
\text { da aplicação de uma avaliação para resolução individual. }\end{array}$ \\
\hline 5 & $\begin{array}{l}\text { Feedback da AAI (Avaliação } \\
\text { Diagnóstica Sistemática) }\end{array}$ & $\begin{array}{l}\text { Etapa dedicada a uma análise conjunta entre docente e discentes, sobre a resolução } \\
\text { da avaliação da etapa anterior. Trata-se de um momento para o autodiagnostico do } \\
\text { aprendizado de cada estudante, focando-se nos domínios cognitivos que apresentaram } \\
\text { desempenho insatisfatório. }\end{array}$ \\
\hline 6 & $\begin{array}{l}\text { Avaliação de Recuperação da } \\
\text { Aprendizagem (ARA) }\end{array}$ & $\begin{array}{l}\text { Etapa dedicada a diagnosticar o nível de aprendizagem real dos estudantes (com } \\
\text { desempenho na etapa } 4 \text { inferior ao mínimo satisfatório) após o processo de } \\
\text { recuperação da aprendizagem realizado na etapa } 5 .\end{array}$ \\
\hline
\end{tabular}

Fonte: elaborado pelo autor.

Figura 3 - Escopo de Execução do Modelo Pedagógico no Período 2

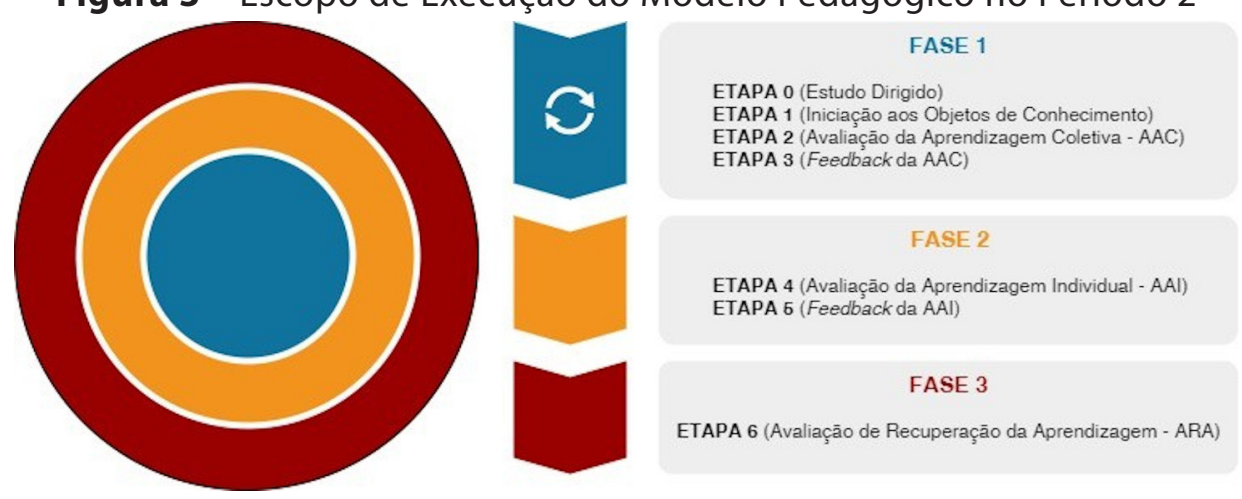

Fonte: elaborado pelo autor.

No Gráfico 4 apresenta-se o desempenho dos estudantes em cada domínio cognitivo e, no Gráfico 5, mostra-se o desempenho médio ponderado. 
Gráfico 4 - Desempenho nos domínios cognitivos no Período 2

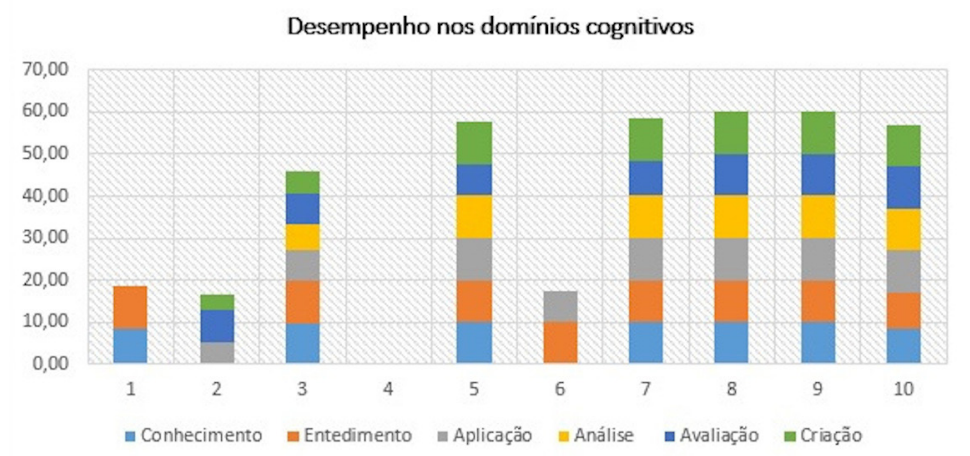

Fonte: elaborado pelo autor.

Gráfico 5 - Desempenho médio ponderado na Etapa 4/Quadro 6 do Período 2

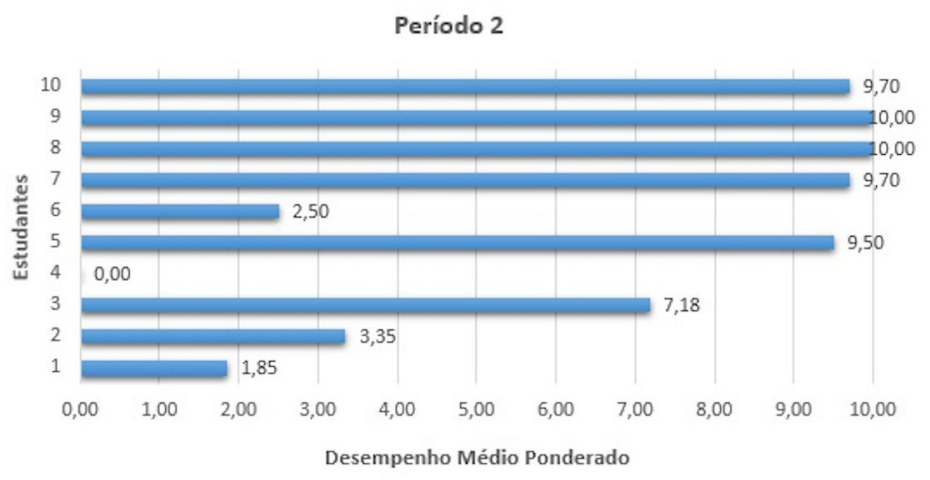

Fonte: elaborado pelo autor.

Observou-se que 4 estudantes não obtiveram o desempenho mínimo satisfatório de 7,0 (benchmark do sistema) e, entre estes, identificou-se baixos níveis de desenvolvimento nos domínios cognitivos tanto da mais alta ordem quanto da mais baixa ordem. Novamente, os estudantes 2, 4 e 6 figuraram entre aqueles com baixo desempenho. Nas avaliações da etapa 2, que permite maior dialogicidade devido à ação de mediação docente, esses estudantes demonstraram déficits de aprendizagem em cálculo diferencial (que confere aos estudantes competências essenciais para o pleno desenvolvimento em Mecânica Geral). Os estudantes 4 e 6 também não apresentaram consistência na assiduidade nos encontros presenciais, devido à compromissos profissionais particulares.

Em relação à desigualdade de aprendizagem entre os grupos que representam a média dos $20 \%$ menores rendimentos e a média dos $20 \%$ maiores rendimentos, constatouse uma desigualdade de $981,08 \%$, onde a mediana do desempenho médio ponderado foi de 8,34 .

Após a Etapa 5 do Quadro 6 - que consistiu no processo de recuperação da aprendizagem - os estudantes com desempenho insatisfatório realizaram uma nova avaliação diagnóstica e os respectivos resultados estão expostos no Gráfico 6. 
Gráfico 6 - Desempenho médio ponderado na Etapa 6/Quadro 6 do Período 1

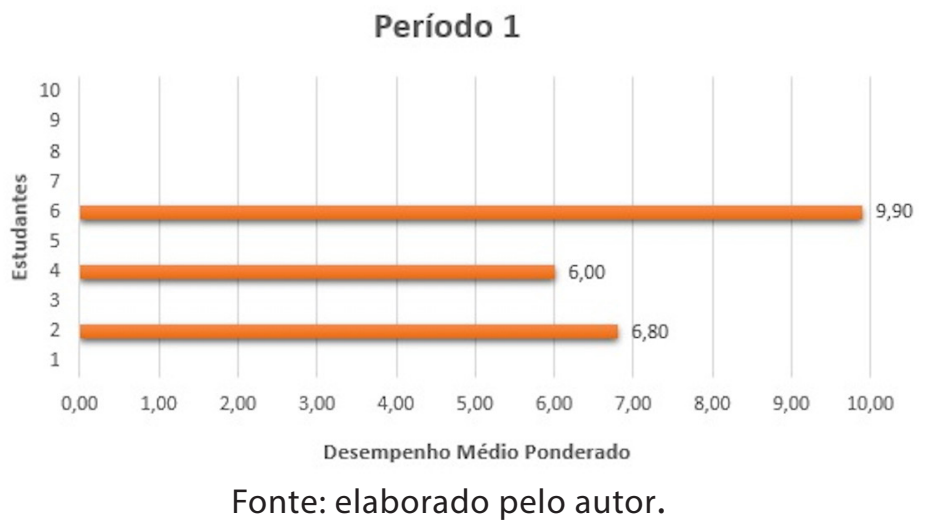

Observou-se que todos os estudantes que realizaram a Avaliação de Recuperação da Aprendizagem (Quadro 6) obtiveram melhoria no desempenho, onde apenas o estudante 6 obteve desempenho acima do mínimo satisfatório. A exceção foi o estudante 1 que optou por não fazê-la.

Também se constatou que a desigualdade de aprendizagem entre os grupos foi de $154,78 \%$ (redução de $84,22 \%$ ), onde a mediana do desempenho médio ponderado foi de 9,60 (aumento de 15,11\%).

Novamente, essas informações evidenciaram que a estratégia de recuperação da aprendizagem também foi eficiente na correção do sistema no período 2, pois maximizou o desempenho cognitivo dos estudantes e também impactou positivamente na queda da desigualdade da aprendizagem entre grupos de estudantes.

\section{Considerações Finais}

Ao longo deste trabalho propôs-se uma metodologia para a aprendizagem ativa fundamentada no realismo ontológico e epistemológico de Mario Bunge.

Ao adaptar o modelo CESM como instrumento para modelagem (análise ex-ante) e validação (análise ex-post) no processo de concepção, execução e avaliação de modelos pedagógicos, dispõe-se aos docentes - responsáveis pela gestão da aprendizagem dos estudantes - uma estrutura lógico-científica coerente com os desafios da educação do século XXI.

No estudo de caso apresentado buscou-se fornecer subsídios de que a utilização do Sistemismo de Bunge, na gestão da aprendizagem ativa, incentiva a utilização de uma abordagem pedagógica baseada em evidências, pois é necessária a implementação das regras metodológicas do Quadro 2, o que corrobora na minimização de vieses cognitivos por parte do docente responsável, induzindo-se a um processo de melhoria contínua dos modelos pedagógicos idealizados.

Neste trabalho optou-se por investigar apenas a aprendizagem cognitiva dos estudantes. Como agenda de pesquisas futuras, sugere-se a incorporação da aprendizagem socioemocional no Modelo CESM, tendo em vista a avaliação do desenvolvimento integral dos estudantes. 


\section{Referências}

AKALIN, S.; SUCUOGLU, B. Effects of classroom management intervention based on teacher training and performance feedback on outcomes of teacher-student dyads in inclusive classrooms. Educational Sciences: theory and practice, Singapore, v. 15, n. 3, p. 739-758, 2015. DOI: https://doi. org/10.12738/estp.2015.3.2543.

ANDERSON, L. W.; KRATHWOHL, D. R. Taxonomy for learning, teaching and assessing: a revision of Bloom's taxonomy of educational objectives. New York: Longman, 2001.

ARANHA, E.; SANTOS, P. H.; GARCIA, N. A. P. EDLE/1: uma ferramenta para o desenvolvimento das habilidades empreendedoras em engenharia. In: CONGRESSO BRASILEIRO DE EDUCAÇÃO EM ENGENHARIA, 45., 2017, Joinville. Anais [...]. Joinville: ABENGE, 2017.

ASYARI, M.; AL MUHDHAR, M. H. I.; SUSILO, H.; IBROHIM, I. Improving critical thinking skills through the integration of problem based learning and group investigation. International Journal for Lesson and Learning Studies, UK, v. 5, n. 1, p. 36-44, 2016. DOI: https://doi.org/10.1108/IJLLS-10-2014-0042.

BADDELEY, A.; EYSENCK, M. W.; ANDERSON, M. C. Memory. 2. ed. London: Psychology Press, $2014 .$.

BONWELL, C. C.; EISON, J. A. Active learning: creating excitement in the classroom. Washington: The George Washington University: School of Education and Human, 1991. (ASHE-ERIC higher education report, 1). Disponível em: https://files.eric.ed.gov/fulltext/ED336049.pdf. Acesso em: 27 set. 2020.

BRASIL. Lei no 9.394, de 20 de dezembro de 1996. Estabelece as diretrizes e bases da educação nacional. Diário Oficial da União, Brasília, p. 27833, 23 dez. 1996.

BUNGE, M. Emergence and convergence: qualitative novelty and the unity of knowledge. Toronto: University of Toronto Press, 2003..

BUNGE, M. Mechanism and explanation. Philosophy of the Social Sciences, UK, v. 27, n. 4, p. 410-465, 1997. DOI: https://doi.org/10.1177/004839319702700402.

BUNGE, M. Systemism: the alternative to individualism and holism. The Journal of Socio-Economics, Dordrecht, v. 29, n. 2, p. 147-157, 2000. DOI: https://doi.org/10.1016/S1053-5357(00)00058-5.

BUNGE, M. A systemic perspective on crime. In: WIKSTRÖM, P.-O. H.; SAMPSON, R. J. The explanation of crime: context, mechanisms and development. Cambridge: Cambridge University Press, 2006. p. 8-30.

CARPENTER, S. K.; CEPEDA, N. J.; ROHRER, D.; KANG, S. H. K.; PASHLER, H. Using spacing to enhance diverse forms of learning: review of recent research and implications for instruction. Educational Psychology Review, UK, v. 24, n. 3, p. 369-378, 2012. DOI: https://doi.org/10.1007/s10648-012-9205-z.

CHIANG, C. L.; LEE, H. The effect of project-based learning on learning motivation and problemsolving ability of vocational high school students. International Journal of Information and Education Technology, UK, v. 6, n. 9, p. 709-712, 2016.

DEMIREL, M.; DAGYAR, M. Effects of problem-based learning on attitude: a meta-analysis study. Eurasia Journal of Mathematics, Science \& Technology Education, UK, v. 12, n. 8, p. 2115-2137, 2016. DOI: https://doi.org/10.12973/eurasia.2016.1293a.

DESLAURIERS, L.; McCARTHY, L. S.; MILLER, K.; CALLAGHAN, K.; KESTIN,G. Measuring actual learning versus feeling of learning in response to being actively engaged in the classroom. Proceedings of the National Academy of Sciences of the United States of America, Washington, v. 116, n. 39, p. 1925119257, 2019. DOI: https://doi.org/10.1073/pnas.1821936116. 
EDUCAUSE. 7 things you should know about flipped classrooms. [S.I.]: Educause, 2012. Disponível em: https://library.educause.edu/-/media/files/library/2012/2/eli7081-pdf.pdf. Acesso em: 28 nov. 2019.

FREEMAN, S.; EDDY, S. L.; McDONOUGH, M.; SMITH, M. K.; OKOROAFOR, H. J.; WENDOROTH, M. $P$. Active learning increases student performance in science, engineering, and mathematics. Proceedings of the National Academy of Sciences of the United States of America, Washington, v. 111, n. 23, p. 8410-8415, 2014. DOI: https://doi.org/10.1073/pnas.1319030111.

INSTITUTO FEDERAL DE EDUCAÇÃO, CIÊNCIA E TECNOLOGIA DE MATO GROSSO DO SUL. Projeto pedagógico de curso: engenharia civil. Aquidauna: IFMS, 2017. Disponível em: https://cutt.ly/ hf1SYdS. Acesso em: 5 nov. 2019.

LO, C. K.; HEW, K. F. Using "first principles of instruction" to design mathematics flipped classroom for underperforming students. International Journal of Learning and Teaching, Spain, v. 3, n. 2, p. 222-236, 2017.

MORETTO, V. P. Planejamento: planejando a educação para o desenvolvimento de competências. Petrópolis: Vozes, 2011.

POPPER, K. R. Textos escolhidos. Rio de Janeiro: Editora PUC-Rio, 2010.

PRINCE, M. Does active learning work?: a review of the research. Journal of Engineering of Education, Hoboken, v. 93, n. 3, p. 223-231, 2004. DOI: https://doi.org/br39.

ROHRER, D.; PASHLER, H. Increasing retention without increasing study time. Current Directions in Psychological Science, UK, v. 16, n. 4, p. 183-186 2007. DOI: https://doi.org/ckrgwd.

SAVERY, J. R. Overview of problem-based learning: definitions and distinctions. Interdisciplinary Journal of Problem-Based Learning, Oklahoma, v. 1, n. 1, p. 9-20, 2006. DOI: https://doi.org/gf3vx9.

UNESCO. Education for sustainable development goals: learning objectives. Paris: Unesco, 2017. Disponível em: https://cutt.ly/ygqMIUO. Acesso em: 25 set. 2020. 